\title{
Th2 cell infiltrations predict neoadjuvant chemotherapy response of estrogen receptor-positive breast cancer
}

\author{
Lan Le ${ }^{1,2 \#}$, Yoshihisa Tokumaru ${ }^{1,3 \#}$, Masanori Oshi ${ }^{1,4}$, Mariko Asaoka ${ }^{1,5}$, Li Yan $^{6}$, Itaru Endo ${ }^{4}$, \\ Takashi Ishikawa ${ }^{5}$, Manabu Futamura ${ }^{3}$, Kazuhiro Yoshida ${ }^{3}$, Kazuaki Takabe ${ }^{1,2,4,5,7}$ \\ ${ }^{1}$ Breast Surgery, Department of Surgical Oncology, Roswell Park Comprehensive Cancer Center, Buffalo, NY, USA; ${ }^{2}$ Department of Surgery, \\ University at Buffalo Jacobs School of Medicine and Biomedical Sciences, The State University of New York, Buffalo, NY, USA; ${ }^{3}$ Department \\ of Surgical Oncology, Graduate School of Medicine, Gifu University, Gifu, Japan; ${ }^{4}$ Department of Gastroenterological Surgery, Yokohama City \\ University, Yokohama, Japan; ${ }^{5}$ Department of Breast Surgery and Oncology, Tokyo Medical University, Tokyo, Japan; ${ }^{6}$ Department of Biostatistics \\ \& Bioinformatics, Roswell Park Cancer Institute, Buffalo, NY, USA; ${ }^{7}$ Department of Surgery, Niigata University Graduate School of Medical and \\ Dental Sciences, Niigata, Japan; ${ }^{8}$ Department of Breast Surgery, Fukushima Medical University School of Medicine, Fukushima, Japan \\ Contributions: (I) Conception and design: L Le, K Takabe; (II) Administrative support: K Takabe; (III) Provision of study materials or patients: K \\ Takabe; (IV) Collection and assembly of data: Y Tokumaru, M Oshi, L Yan; (V) Data analysis and interpretation: M Asaoka, I Endo, T Ishikawa, M \\ Futamura, K Yoshida; (VI) Manuscript writing: All authors; (VII) Final approval of manuscript: All authors. \\ \#These authors contributed equally to this work. \\ Correspondence to: Kazuaki Takabe, MD, PhD, FACS. Breast Surgery, Department of Surgical Oncology, Roswell Park Comprehensive Cancer \\ Center, Elm \& Carlton Streets, Buffalo, NY 14263, USA. Email: kazuaki.takabe@roswellpark.org.
}

Background: High infiltration of Th2 is linked to breast cancer progression and metastasis through the induction of cytokine release and T-cell anergy. The estrogen receptor (ER)-positive subtype, which accounts for $70 \%$ of breast cancer, is known to respond less to neoadjuvant chemotherapy (NAC) due to its low potential for proliferation. We hypothesized that Th2 high tumors are highly proliferative, and thus more likely to respond to NAC in ER-positive breast cancer.

Methods: We obtained clinicopathological data and overall survival information on 1,069 breast cancer patients from The Cancer Genome Atlas (TCGA). Computational algorithms and CIBERSORT were used to estimate immune cell infiltration. Additionally, xCell was used for validation.

Results: Th2 high tumors did not consistently associate with an unfavorable immune cell composition and tumor immune microenvironment but were found to be significantly elevated in the cancer stage. Th2 high tumors also correlated with high Nottingham pathological grade, as well as with Ki-67 and proliferation score in ER-positive subtypes. High Th2 tumors achieved a pathological complete response (pCR) significantly higher in ER-positive breast cancer.

Conclusions: In conclusion, high levels of Th2 are associated with aggressive features of breast cancer. Th2 levels may be a biomarker in patient selection for NAC in ER-positive breast cancer.

Koywords: Th2; type 2 helper T cells; tumor immune microenvironment; breast cancer; ER positive

Submitted Jun 20, 2020. Accepted for publication Oct 30, 2020.

doi: $10.21037 /$ gs-20-571

View this article at: http://dx.doi.org/10.21037/gs-20-571

$\wedge$ ORCID: 0000-0002-4727-8005. 


\section{Introduction}

The tumor immune microenvironment has been of interest in recent research because we now know the dual role of the immune system in cancer: it can not only suppress tumor growth but also promote tumor progression (1). Given the complexity of the immune system as a whole, prior studies have found that different cell types lead to cancer progression by induction of T-lymphocytes and cancer suppression by the polarization of these same lymphocytes into different subgroups. Specifically, CD4+ T-cells can differentiate into Th1 or Th2 helper T-cells which leads to regression versus promotion of tumor (2).

Th2 cells are known to promote tumor progression through the secretion of cytokines IL-4, IL-6, IL-10, and IL-13 to activate tumor-associated M2 macrophages (2). Activation of these M2 macrophages in part stimulates tumor progression by promoting tumor cell growth and invasion as their activation can enhance angiogenesis. In addition, TGF- $\beta$ which is normally released by Th17 cells and T-regulatory cells (T-regs), is a cytokine known to attenuate the immune response. Increased expression of TGF- $\beta$ has been associated with malignant conversion and progression in breast cancer as well as gastric, endometrial, ovarian, gliomas, and melanomas (3).

On the contrary, CD4+ T-cells can differentiate into Th1 cells to promote tumor regression. Th1 induced responses have been described to be beneficial in antitumor immunity. They secrete IFN- $\gamma$ and many other cytokines to induce activation of $\mathrm{M} 1$ tumor-associated macrophages and cytotoxic CD8+ T-cells (2).

It is known that proliferative cancer cells respond better to neoadjuvant chemotherapy (NAC). Proliferation can be assessed by Ki-67 or mitotic index, and increased proliferation has been shown to correlate with poor prognosis (4). Triple-negative breast cancer (TNBC), which lacks expression of estrogen receptor (ER), progesterone receptor (PR), and human epidermal growth factor receptor 2 (HER2), are highly proliferative. Thus, they are more likely to respond to NAC than the ER-positive subtype $(5,6)$. Therefore, we speculated that highly proliferative ER-positive breast cancer may respond to NAC better than lower proliferative ones.

Recently our group has been analyzing the tumor immune microenvironment with computational algorithms on transcriptomes (7-14). We utilized the publicly available patient cohorts that have gene expression data linked to clinical data. We then applied computational algorithms to estimate the immune cell infiltration of interest. In this study, we hypothesized that high infiltration of Th2 cells is associated with aggressive features of breast cancer. If that is the case, we further hypothesize that Th2 expression level may be a possible predictive biomarker of NAC.

We present the following article in accordance with the MDAR reporting checklist (available at http://dx.doi. org/10.21037/gs-20-571).

\section{Methods}

\section{Data acquisition of The Cancer Genome Atlas (TCGA)}

Clinicopathological data and overall survival information were obtained on 1,069 breast cancer patients from TCGA. This is publicly available data provided by the National Cancer Institute and the National Human Genome Research Institute. TCGA was downloaded utilizing cBioportal as described previously (10-13,15-21). GSE25066 and GSE23988 are the cohorts of patients that underwent NAC with Taxane and anthracycline-based regimen $(\mathrm{n}=502)$ and FEC (5-Fluorouracil, Epirubicin, Cyclophosphamide) with Taxane regimen $(\mathrm{n}=61)$. We defined Th2 high and low levels with the median cutoff (12). Clinicopathological demographics of the TCGA cohort were stratified into Th2 high and Th2 low groups. The Institutional Review Board (IRB) approval was waived as this entire study was conducted on deidentified publicly available data sets provided by TCGA.

\section{Estimation of the immune cell composition, scores, intratumoral heterogeneity, neoantigens, mutation rates and homologous recombination defects}

Thorsson et al.'s published pre-calculated estimates of the immune cell composition of the patients in TCGA were used for the current analysis (22). CIBERSORT, a deconvolution algorithm published in Nature Methods in 2015 by Newman et al. (23) was used in Thorsson's analysis (22). By utilizing transcriptome data, this algorithm enables us to estimate the fraction of 22 immune cell types in each bulk tumor $(9-12,24,25)$. The estimated immune cell composition was validated using the xCell algorithm on TCGA cohorts (14). Likewise, TGF- $\beta$ response score and IFN- $\gamma$ response score, as well as cell proliferation and aneuploidy score, were obtained from the same source (22). Further Intratumor heterogeneity, SNV and 
Indel Neoantigens, Silent and Nonsilent Mutation Rates, Number of Segments, Fractional Altered, and Homologous Recombination Defects data were all obtained from the same source with additional analyses using $x$ Cell and Thorsson et al.'s analysis (22).

\section{Gene set enrichment analysis (GSEA)}

GSEA was performed comparing high and low Th2 expressing tumors among hallmark gene sets using software provided by the Broad Institute (http://software. broadinstitute.org/gsea/index.jsp), as we described previously (11-13,17,21,26-28).

\section{Statistical analysis}

Statistical analyses were performed using $\mathrm{R}$ software (version 3.6, http:///www.r-project.org/) and Bioconductor (http://bioconductor.org/). The differences between the two groups were assessed using the Fisher exact test for categorical variables. A two-sided $\mathrm{P}<0.05$ was considered statistically significant. Correlation analysis was performed using the Spearman method.

\section{Results}

Th2 bigh breast cancer was associated with $T$ and $N$ category of cancer staging, Her 2 receptor positivity, as well as infltrating ductal and tubular carcinoma

The association between Th2 expression levels and clinicopathological features of breast cancer from the TCGA was analyzed. Patients were stratified into Th2 high and Th2 low groups using a median cutoff. Th2 high groups were significantly associated with cancer staging, especially the $\mathrm{T}$ and $\mathrm{N}$ category but not the $\mathrm{M}$ category. Th2 high tumors were statistically significant with Her2 receptor positivity. Histological subtypes such as infiltrating ductal and tubular carcinoma showed statistical significance by Th2 expression levels (Table S1).

\section{Th2 bigh breast cancer did not consistently associate with unfavorable tumor immune microenvironment}

The current conception is that Th2 cell infiltration inhibits cytolytic lymphocytes and create an unfavorable tumor immune microenvironment that leads to tumor promotion and suppression of the immune cells that support tumor regression (29). We used human breast cancer cohorts with gene expression profile (TCGA) to investigate this current concept in human breast cancer. As expected, tumorpromoting M2 macrophages were significantly infiltrated in Th2 high tumors. However, T-regs were inconsistently infiltrated in the Th2 high groups with significance seen only in Thorsson et al.'s analysis $(\mathrm{P}<0.001)$ and not in analysis using $x$ Cell $(\mathrm{P}=0.803$; Figure 1).

Similarly, we suspected that tumor regressing immune cells would be less infiltrated in Th2 high tumors. On the contrary, we found that tumor regressing M1 macrophages were highly infiltrated in Th2 high tumor groups $(\mathrm{P}=0.01$; Figure 1$)$. These calculations were validated by our calculations using the $x$ Cell algorithm $(\mathrm{P}<0.001$; Figure 1B) (24). We found that Th2 high tumors were not consistently associated with the enhancement of an unfavorable tumor immune microenvironment nor suppression of a favorable tumor immune microenvironment, as previously postulated in the TCGA cohort.

Cytokines IL-4, IL-6, IL-10, and IL-13, as well as TGF- $\beta$ and IFN- $\gamma$ response scores did not associate unfavorably with Th2 high tumors

Th2 cell is known to release acute phase cytokines such as interleukins IL-4, IL-6, IL-10, and IL-13 when appropriately activated by $\mathrm{CD} 4^{+} \mathrm{T}$-cells (29). The expression of these cytokines is reported to induce T-cell anergy and loss of T-cell-mediated cytotoxicity (29). We investigated the expression of these cytokines in human breast cancer and found that the Th2 expression levels did not correlate to IL-4, IL-6, IL-10 and IL-13 levels ( $\mathrm{r}=-$ $0.063, \mathrm{r}=-0.127, \mathrm{r}=0.059, \mathrm{r}=-0.009$ respectively) (Figure $2 A$ ).

Further, Th2 high tumors were reported to associate with TGF- $\beta$ secretion, which supports tumor promotion, and with suppression of IFN- $\gamma$, which regress tumors (2). We found that the TGF- $\beta$ response score was significantly low $(\mathrm{P}<0.001)$, and the IFN-y response score was significantly high on Th2 high tumors $(\mathrm{P}<0.013)$, which was opposite to what we expected (Figure $2 B$ ). Again, Th2 high tumors were not associated with an unfavorable tumor immune microenvironment in the TCGA breast cancer cohort.

\section{Th2 levels were associated with cancer staging}

Based on the previous reports by DeNardo et al. we assumed that Th2 high breast cancers have aggressive features (2). To see its association with a clinical parameter, we investigated 
A
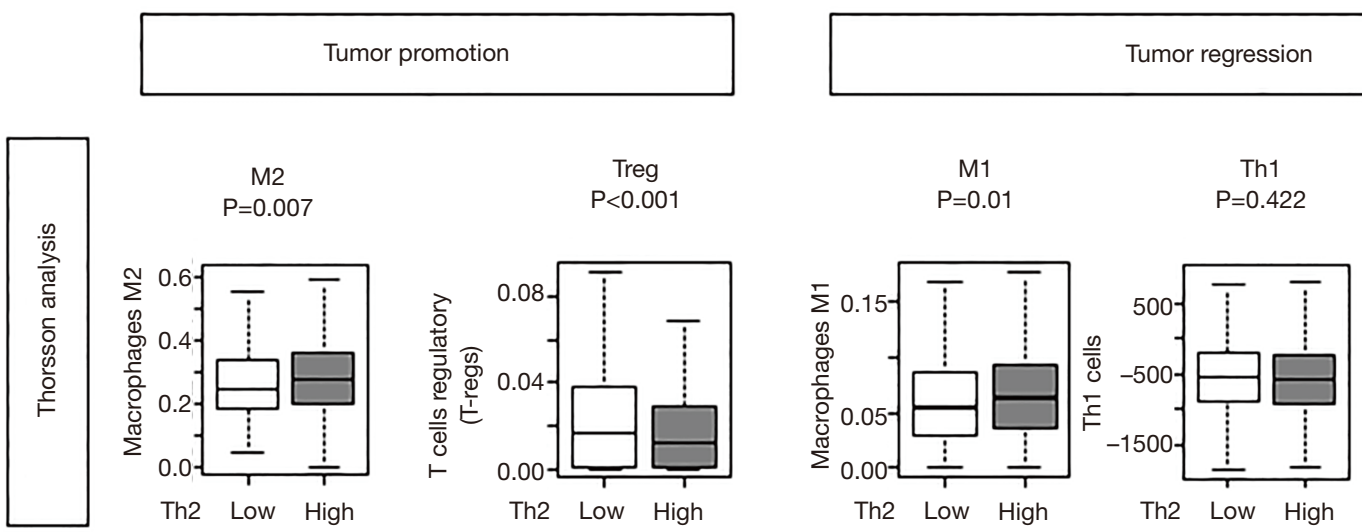

Tumor regression

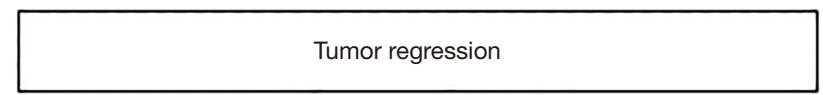

B
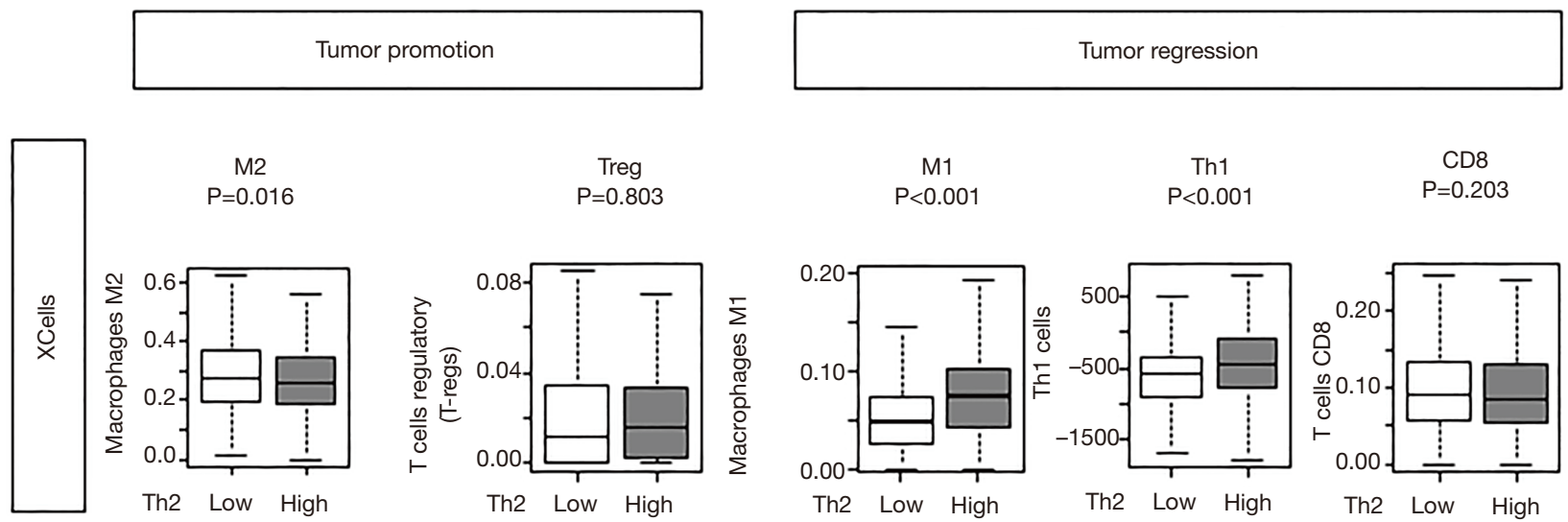

Figure 1 Th2 high tumor groups did not consistently associate with unfavorable tumor immune microenvironment. (A) Thorsson analysis of tumor promoter cells [M2, T regulatory (T-reg) cells] and tumor regressor cells (M1, Th1 and CD8). M2 macrophages were found to be highly infiltrated in Th2 high tumors but T-regs were found to not be highly infiltrated in Th2 high tumors. (B) xCell algorithm analysis of tumor promoter cells (M2, T-reg cells) and tumor regressor cells (M1, Th1 and CD8). M1, Th1 and CD8 were also found to be highly infiltrating in Th2 high tumors.

the Th2 levels based upon the American Joint Commission on Cancer (AJCC) cancer staging that consists of $\mathrm{T}$ (primary tumor), $\mathrm{N}$ (regional lymph node), and $\mathrm{M}$ (distant metastasis) categories. Analysis with ANOVA showed there was a significant elevation of Th2 levels by clinical features of stage $(\mathrm{P}<0.005)$ except for the $M$ category (Figure $3 A)$.

\section{Th2 levels correlate with the Nottingham pathological grade in ER-positive breast cancer}

Given the association with cancer staging, we hypothesized that the Th2 levels correlate with the cancer aggressiveness measured by the Nottingham pathological grade, which consists of tumor histological scores for mitotic rate, nuclear grade, and tubular score. Th2 level was found to have a significant correlation with high mitotic and nuclear score, but not with tubular score in the whole cohort $(\mathrm{P}<0.001)$ (Figure $\mathrm{S} 1 \mathrm{~A})$. When we performed a similar analysis for the ER-positive subtype, we found that there was a significant correlation of Th2 levels with pathological grade, particularly with the mitotic and nuclear score (all $\mathrm{P}<0.001$ ), but not tubular score (Figure $3 B$ ). Interestingly, there was no correlation in the TNBC, which may be because the majority of them had a high score.

\section{Th2 levels correlated with a cell proliferation marker, Ki- 67, in ER-positive breast cancer}

Given the correlation of Th2 levels and the pathological grade, we further hypothesized that Th2 levels correlate 
A

IL-4 $r=-0.063(P=0.004)$

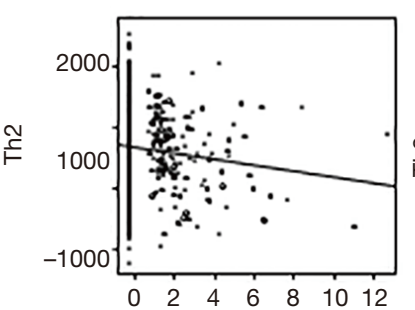

IL-6 $r=-0.127(P<0.01)$

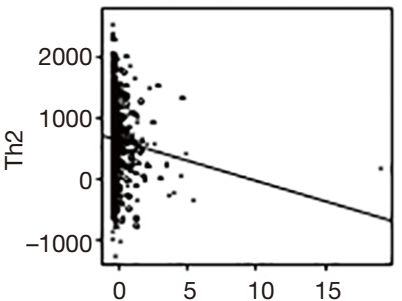

B

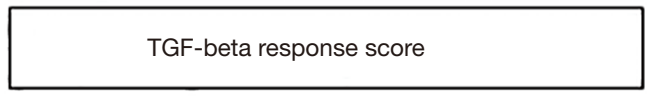

$\mathrm{P}<0.001$

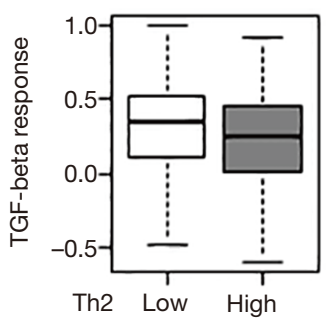

IL-10

$r=0.059(P=0.05)$

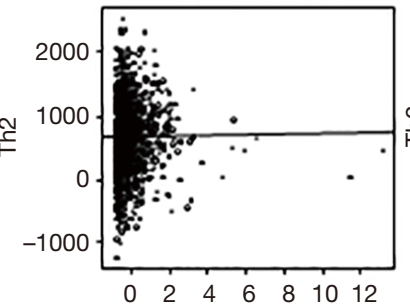

IL-13

$$
r=-0.009(P=0.77)
$$

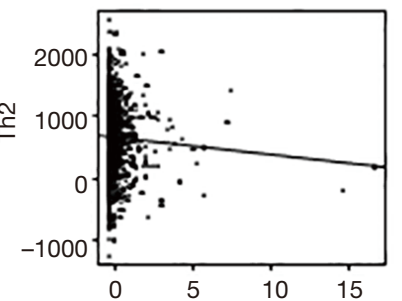

IFN-gamma response score

$\mathrm{P}<0.013$

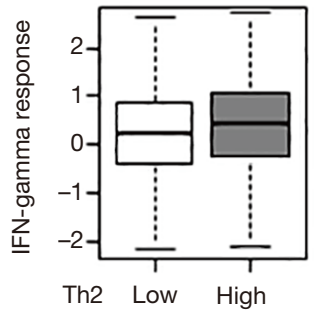

Figure 2 Th2 expression levels correlated with immune related response scores but did not with Th2 associated cytokines. (A) Th2 expression levels did not correlate with IL-4, IL-6, IL-10, and IL-13, which were known to be associated with an unfavorable tumor immune microenvironment (B) Th2 high tumors demonstrated low TGF- $\beta$ response score in the Thorsson analysis. Th2 high tumors demonstrated high IFN- $\gamma$ response score in the Thorsson analysis.

with the most commonly used cell proliferation marker, $\mathrm{Ki}-67$, which is a nuclear protein expressed in cell cycle phase S, G1, G2, and M (30). We found that there was a high correlation of Th2 levels with Ki-67 $(r=0.679)$ as well as proliferation score $(r=0.676)$ in the whole cohort (Figure S1B). We did the same analysis on the ER-positive subtype and found that Ki-67 and proliferation scores were significantly correlated with Th2 expression levels (Figure $3 C ; \mathrm{r}=0.74$ and $\mathrm{r}=0.759$, respectively). When we performed the same analysis for TNBC, we found that the correlation of Th2 expression levels and Ki-67 or proliferation score was not as strong as the ER-positive subtype (Figure $3 C ; \mathrm{r}=0.548$ and $\mathrm{r}=0.674$, respectively).

\section{Th2 bigh breast cancer enriched the gene sets related to cell cycle and cell proliferation}

Gene set enrichment analysis allows us to estimate the underlying mechanism of the gene expression profile. We found that the following gene sets were enriched in Th2 high tumors; G2M checkpoint, mitotic spindle change, E2F targets, PI3K/AKT/MTOR signaling pathway, and MTORC1 signaling pathway, all related to cell cycle and cell proliferation $(\mathrm{P}<0.001)$. Enrichment plots for these gene sets are shown in Figure 4. Taken together with pathological analyses, $\mathrm{Ki}-67$, and proliferation scores, as well as gene set analyses, Th2 high breast cancer, is associated with enhanced cancer cell proliferation.

\section{Th2 high breast cancer was associated with higher intratumor heterogeneity, homologous recombination defect, and mutation load, but not with neoantigen}

Given its association with tumor aggressiveness, it was of interest to see what underlying tumor features associate with high infiltration of Th2. Currently, it is thought that a high tumor mutation burden created by multiple mechanisms, including homologous recombination defect, which generates neoantigens, attracts immune cells to the tumor microenvironment. Utilizing the precalculated data 
A
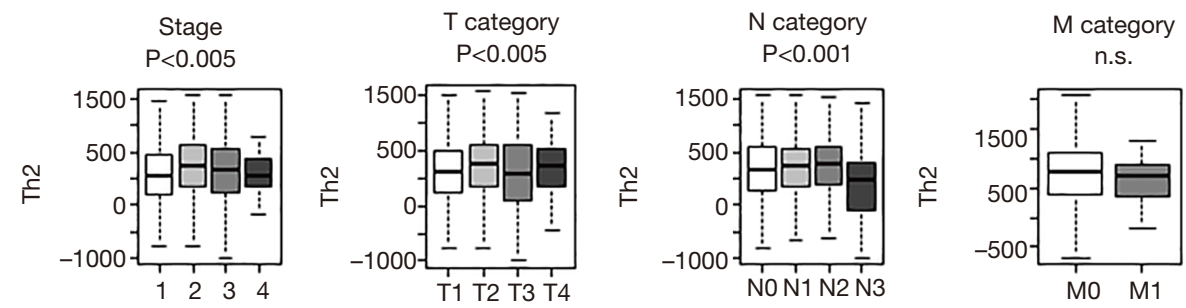

B
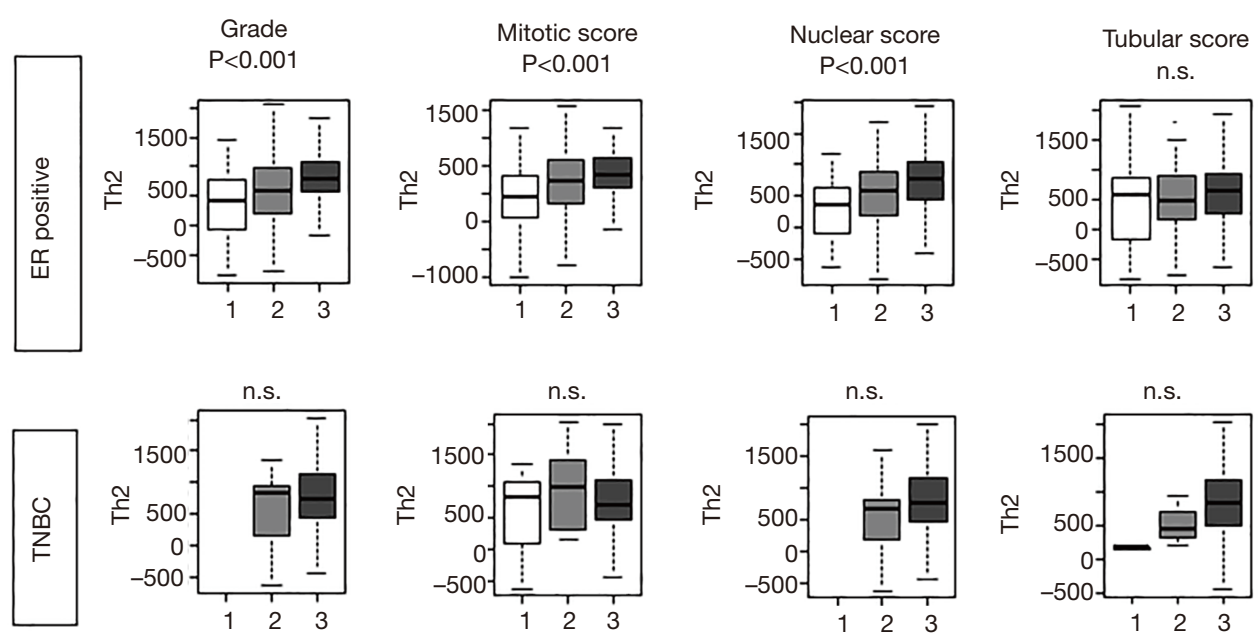

C
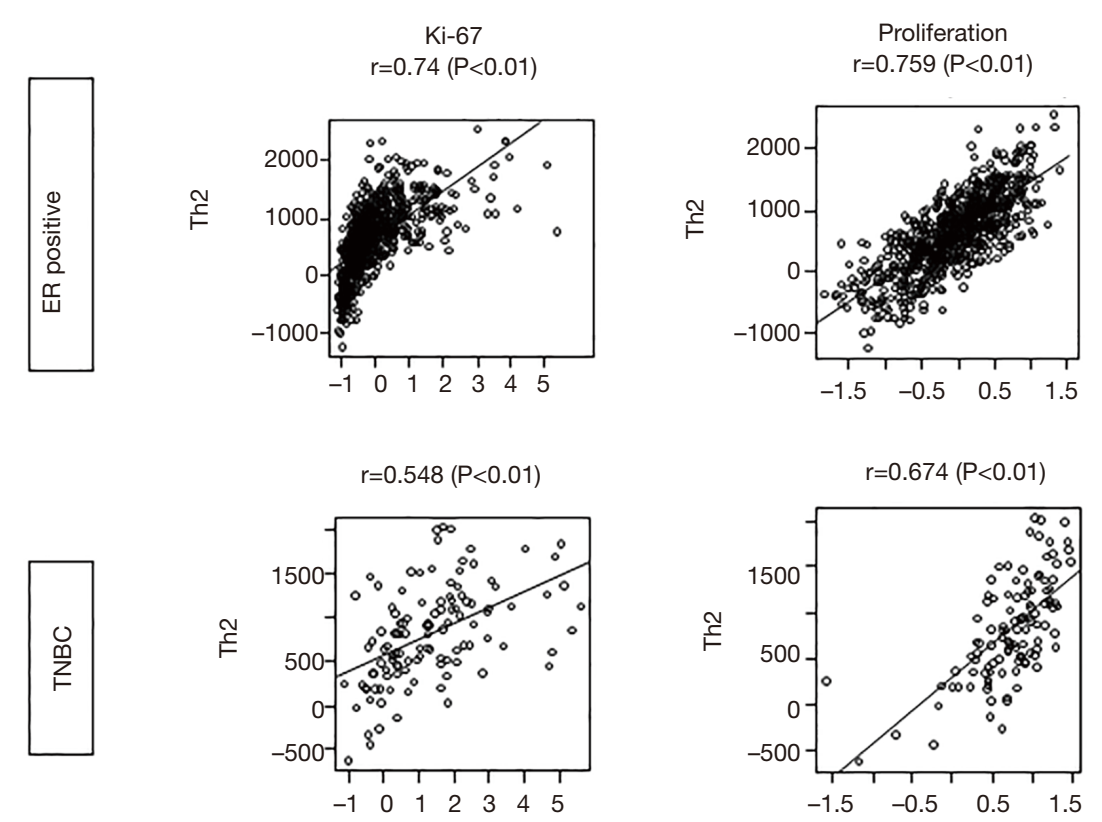

Figure 3 Th2 expression levels are associated cancer staging, pathological grade, especially mitotic and nuclear score, but not tubular score. Th2 high tumor groups correlated with Ki-67 and proliferation score. (A) Analysis showed a significant elevation of Th2 levels by cancer stage. (B) ER-positive breast cancer groups showed significant correlation of Th2 expression levels to pathological grade, especially with the mitotic and nuclear score, but not tubular score. These results were not seen in TNBC with no significance in overall grade, mitotic, nuclear and tubular score. (C) Th2 high, ER-positive breast cancer showed significant correlation with Ki-67 nuclear protein and proliferation score. There was only moderate correlation seen in Th2 high TNBC. ER, estrogen receptor; TNBC, triple-negative breast cancer. 

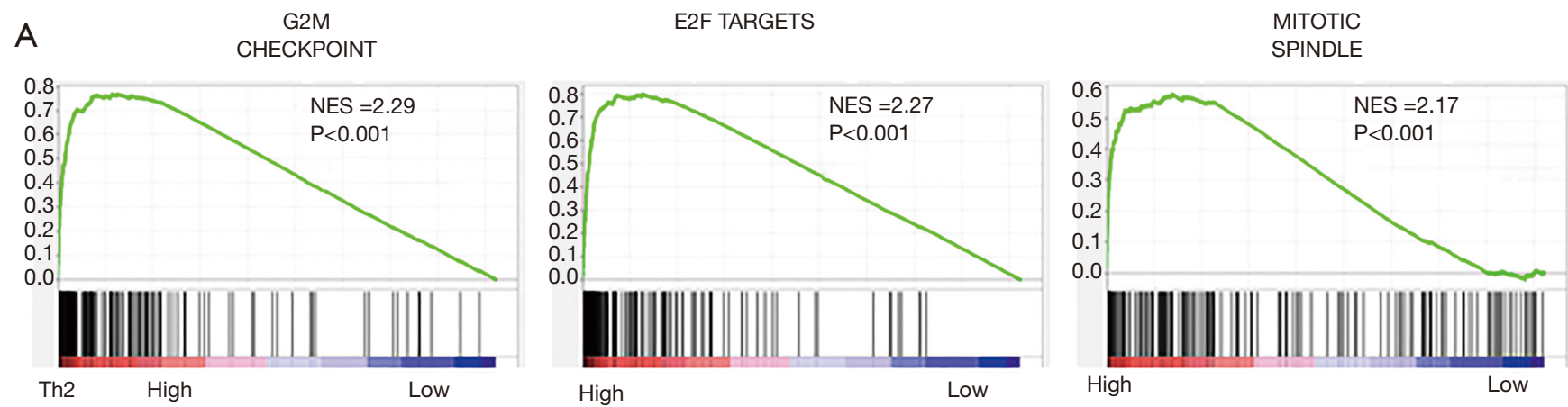
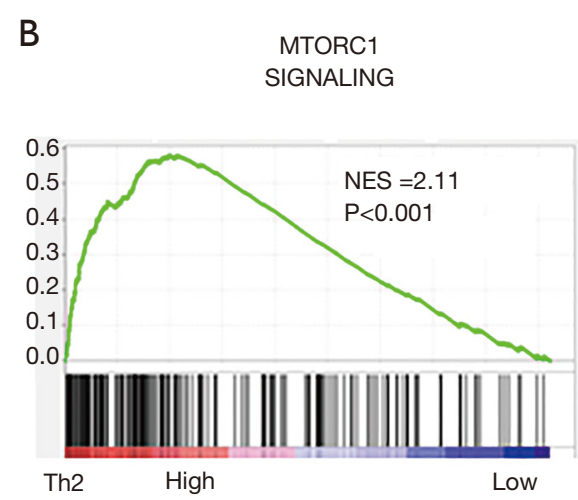

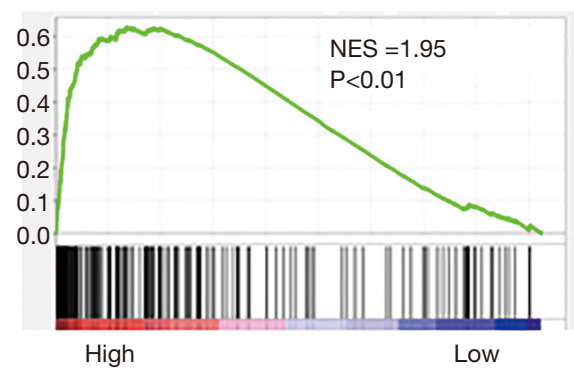

High
MYC TARGETS

V1

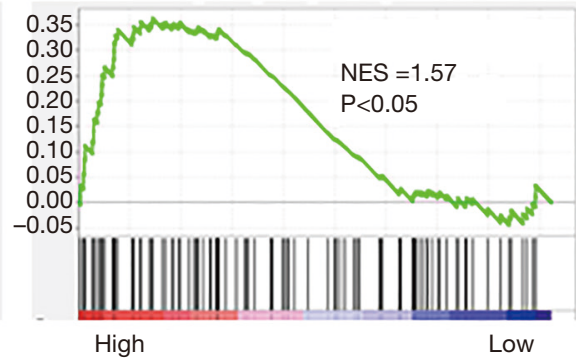

Figure 4 Th2 high breast cancer enriched the gene sets related to cell cycle and cell proliferation. These are enrichment plots depict hallmark gene sets enriched in the Th2 high groups (G2M checkpoint, E2F Targets, Mitotic spindle, MTORC1 signaling, MYC Targets V1, PI3K/AKT/MTOR signaling).

published by Thorsson et al. we found that intratumoral heterogeneity was significantly higher in Th2 high breast cancer $(\mathrm{P}<0.001$; Figure 5$)$. Mutation measurements such as the number of segments, altered fractions, aneuploidy scores were also significantly higher in Th2 high breast cancer (all $\mathrm{P}<0.001)$. The homologous recombination defect in Th2 breast cancer implicates the gnomically unstable cancer which is also associated with high Th2 cell infiltration. However, neoantigen load (SNV and Indel neoantigen and silent and nonsilent mutation rate), were found to be no different by Th2 levels.

\section{Th2 high breast cancer was associated with pathological complete response ( $p C R$ ) to $\mathrm{NAC}$ in ER-positive breast cancer}

It is well known that only a few ER-positive breast cancer patients respond to NAC compared to TNBC. Therefore, any biomarker to identify the ER-positive breast cancer that responds to NAC will be of benefit in patient selection.
We identified two cohorts (GSE25066, n=502, treated with Taxane and anthracycline-based regimen and GSE23988, $\mathrm{n}=61$, treated with FEC (5-Fluorouracil, Epirubicin, Cyclophosphamide) and Taxane regimen) with gene expression data associated with response to NAC (Figure 6). First, we found that the patients who achieved pCR have significantly higher Th2 levels compared with the ones who had the residual disease (RD) (Figure S2). This led us to investigate whether the Th2 high tumors were associated with a significantly high ratio of $\mathrm{pCR}$, which was the case (Figure 6). Interestingly, this was only seen in ER-positive and not ER negative subtypes. This result suggests that a high level of Th2 cells can be a predictive biomarker of ERpositive breast cancer to achieve pCR after NAC.

\section{Discussion}

It has been postulated that high infiltration of Th2 cells associated with aggressive cancer by promoting an unfavorable tumor immune microenvironment. 

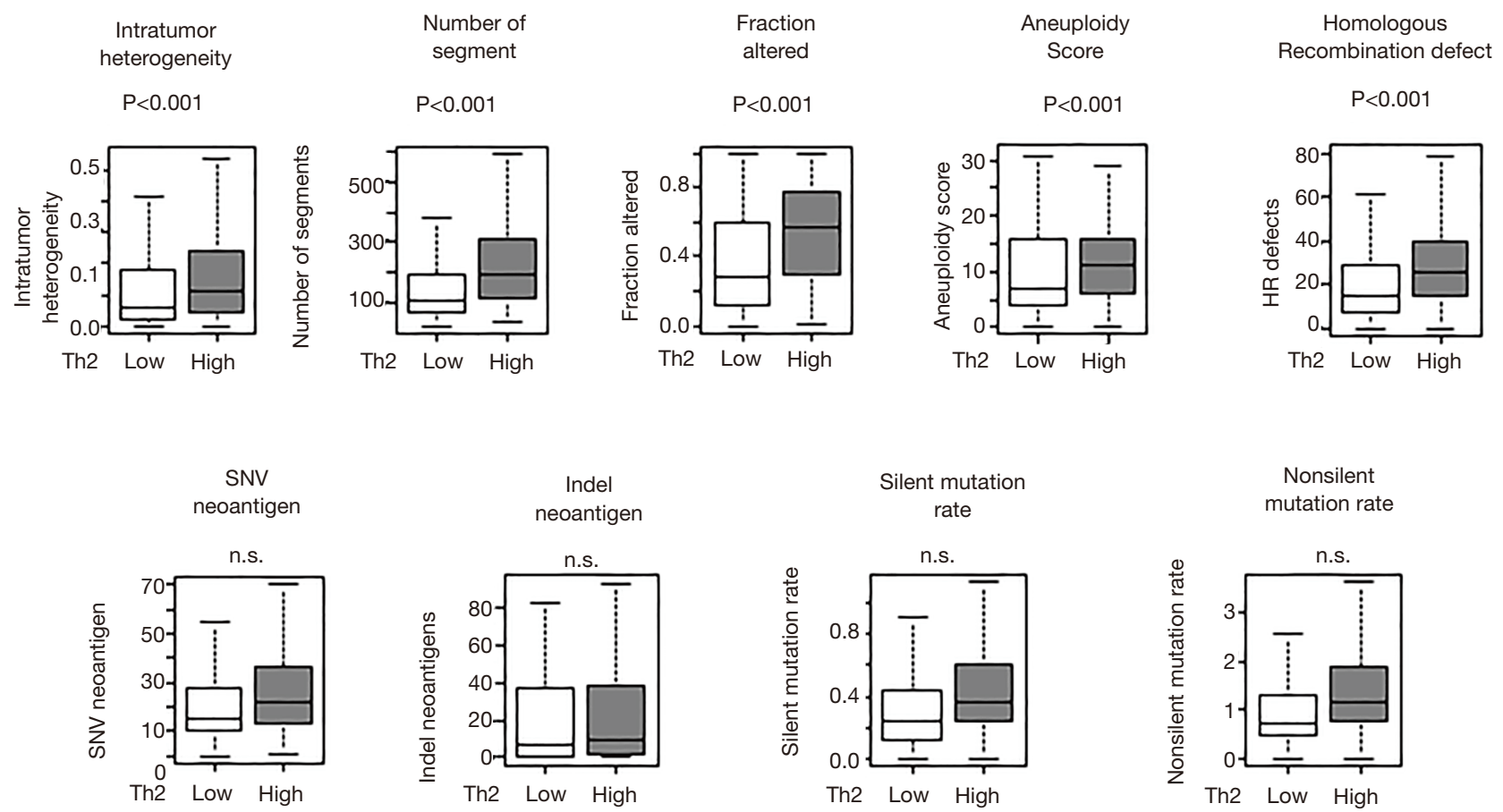

Figure 5 Th2 high breast cancer was associated with higher Intratumor Heterogeneity, but not with neoantigen load or mutation rate. This is the $\mathrm{xCell}$ analysis of intratumoral heterogeneity and mutation measurements (number of segments, altered fractions, aneuploidy score, silent and nonsilent mutation rates) as well as neoantigen load.

A

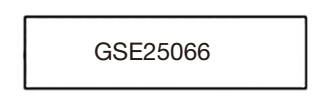

ER+

$\mathrm{P}<0.05$

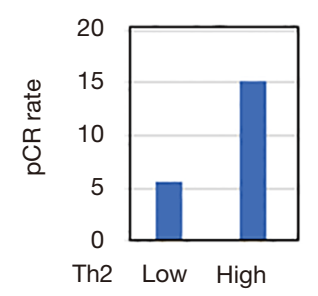

B

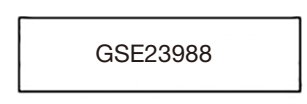

$$
\text { ER+ }
$$

$\mathrm{P}=0.083$

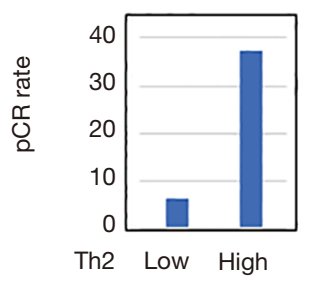

ER$P=0.462$

Figure 6 Th2 high breast cancer was associated with pathological complete response (pCR) to neoadjuvant chemotherapy (NAC) in ERpositive breast cancer. Analysis of two cohorts (GSE25066 and GSE23988) with gene expression data showed patients who achieved pCR had significantly higher Th2 levels and that Th2 high tumors demonstrated high ratio of pCR in estrogen receptor (ER)-positive breast cancer patients. 
In the TCGA human breast cancer cohort high Th2 cells associated with increased tumor promoting M2 macrophages; however, tumor-regressing immune cells such as M1 macrophages, Th1, and CD8 cells were also increased, which contradicted the earlier report. Th2 levels also did not correlate with any interleukins that induce activation of the tumor promoting M2 macrophages, and an opposite trend was seen in TGF- $\beta$ and IFN- $\gamma$ responses. On the other hand, Th2 levels did significantly associate with aggressive cancer features seen clinically in cancer stage, pathologically by Nottingham grade and molecular biologically in $\mathrm{Ki}-67$ expression, proliferation score in ER-positive, but not in TNBC. GSEA enriched cell proliferation related gene sets such as G2M checkpoint, E2F Targets, Mitotic Spindle, MYC Targets, mTORC1 signaling, and PI3K/AKT/mTOR signaling. Additionally, we found that high intratumoral heterogeneity, homologous recombination defect, and mutation load, but not neoantigen associated with high Th2 cells. Finally, we found that Th2 levels were significantly higher in pCR patients and the pCR rate was significantly high in Th2 high tumors in ER-positive and not in TNBC.

Coussens $\mathrm{et} \mathrm{al}$. previously postulated the paradoxical roles of the immune system in cancer progression. Activation of immune cells can lead to tumor promotion or regression through complex mechanisms in the tumor immune microenvironment (31), which is to be linked to the polarity of CD4+ T-helper cells (2). The favorable tumor immune microenvironment consists of CD4+ T-cells which when stimulated, differentiate into anti-tumor Th1 helper cells that release cytokines such as IFN- $\gamma$ to collaborate with M1 tumor-associated macrophages and the cytotoxic functions of T-lymphocytes (2). In contrast to this, Th2 differentiated helper cells release cytokines IL-4, IL-6, IL-10, and IL-13 that stimulate tumor-associated M2 macrophages, leading to tumor promotion.

M2 macrophages are known to enforce tumor promotion by suppressing cytotoxic T-lymphocytes. Additionally, through innate signals, M2 macrophages induce T cells into T-regulatory (T-reg) cells (32). T-regs secrete TGF- $\beta$ and have potent immunosuppressive activities that counteract the anti-tumor response and favor tumor promotion (33). In our study, we identified M2 macrophages as significantly infiltrated in Th2 high tumor groups. However, we were unable to find consistent significance in T-reg infiltration. This may be due to T-reg's unknown mechanism of accumulation and expansion. Additionally, T-regs have postulated to exert opposite functions depending on the tumor type. Though this is still under investigation, it is plausible that the phenotypical uniqueness of each tumor immune microenvironment may recruit different Treg subtypes (33).

We were surprised to find that Th2 high tumors were not associated with Th2-related cytokines and were not associated with TGF-beta and IFN gamma signatures in the way of the earlier reports. However, considering that our study is the first to investigate this association in a large human breast cancer patient cohort and are not repeating the same experiments of the earlier reports, the mechanisms proved in cell cultures and animal experimental settings may not be applicable in the patients. Further, we have recently reported that tumor promotion/regression signatures are not always mutually exclusive. Although M1 and M2 macrophages commonly show the opposite trend $(18,34)$, we found that both were significantly decreased simultaneously in intratumoral adipocyte-high breast cancer (35).

The gold standard to analyze tumor immune microenvironment is flow cytometry of fresh tissue samples. Occasionally immunohistochemistry of fixed slides has been used, however, quantification of pathological analyses was known to be inaccurate. Although it proves value in basic science research, their utility in a large sample size for clinical patients can be challenging given the limited access to fresh samples, cost, and labor. Thus, to overcome this difficulty we have defined Th2 following the methods reported by Thorsson (22) and validated the results using $x$ Cell. It was not our intention to prove that Th2, defined by the Thorsson method, is identical to Th2 cells detected by the gold standard. Our intention was to introduce this modern technology of in Silico computational biology to the field that allows analyses of multiple large patient cohorts and allow investigation on clinical relevance when we define cells by transcriptome. One of the reasons we chose this method was because the original article that reported the Thorsson method (22) has been cited 766 times in 2 years after its publication and is now becoming a new standard. To this end, our main goal was to show the clinical relevance of Th2 cell levels defined by the Thorsson method rather than to validate whether the Thorsson analysis reproduces the gold standard.

We have reported that tumors with high mutation rates demonstrated aggressive phenotypes with highly proliferative features, while simultaneously attracting anti-tumor immune cells in the tumor immune microenvironment with high immune activity such as 
IFN- $\gamma$ response (34). We found in the current study that high Th2 cells were associated with high mutation load as well as infiltration of anti-tumor cells such as Th1 cells and $\mathrm{CD} 8$ cells and high IFN- $\gamma$ response score. Since this is a retrospective analysis of the transcriptome, we have no data on causality. And although theoretically, Th2 cells may have some effect on clonal selection, given that there is no report on such a function, it is more likely that Th2 cell infiltration is a result of high mutation load. If that is the case, we cannot help but speculate that high infiltration of tumorpromoting immune cells may be due to the function of Th2 cells and high infiltration of tumor-regressing immune cells may be the result of attraction by high mutation load, and that is the reason why we see both types of immune cells in high Th2 cell tumors.

We have previously reported that intratumoral heterogeneity may account for the difference in tumor behavior with a unique tumor immune microenvironment (16). This study found that high mutantallele tumor heterogeneity, which is a bioinformatic algorithm that provides a measurable and quantitative assessment of intratumor heterogeneity, was associated with significantly higher fractions of immunosuppressive T-regs. In the current study, we found that intratumoral heterogeneity was significantly associated with Th2 high tumor although infiltration of T-regs was inconsistent by the analyses.

Interestingly, this association of Th2 cell infiltration and cancer aggressiveness was only apparent in ER-positive and not in TNBC. In both pathological analyses using grade and molecular biological analyses using Ki-67 expression and proliferation score, Th2 high ER-positive breast cancer had statistically significant associations. From the data, it appears that all TNBC are highly proliferative at baseline. Thus, Th2 levels may have no impact on the aggressiveness of TNBC. Whereas in the less proliferative ER-positive subtype, there is a significance. Taken together, Th2 cell infiltration may be a marker for cancer aggressiveness by mutation load and/or intratumoral heterogeneity in the ERpositive subtype.

One of the goals of NAC is to improve surgical outcomes by shrinking the tumor. It is well known that the majority of ER-positive breast cancer does not respond to NAC; thus, it is often not even offered. In our study, Th2 high tumors demonstrated a high ratio of PCR, which is a most likely reflection of our results that the Th2 cell level is a marker of highly proliferative cancer. Thus, we cannot help but speculate that Th2 may be a good predictive biomarker for highly proliferative ER-positive breast cancer that responds to NAC.

This study has its limitations. Data were obtained through publicly available data sets such as the TCGA, representing a retrospective study. To establish Th2 as a possible predictive biomarker for NAC, we would need to perform a prospective study. Furthermore, we are unable to definitively conclude whether Th2 levels are high due to the proliferative nature of certain breast cancers, or high Th2 levels are causing these proliferative features of breast cancer. To clarify the causality, an experimental study is needed. However, the focus of this study is to understand the clinical significance of highly proliferative Th2 tumor groups.

\section{Conclusions}

In conclusion, high levels of Th2 are associated with aggressive features of breast cancer although it was not consistently associated with unfavorable tumor immune microenvironment. Th2 levels may be a biomarker in patient selection for NAC in ER-positive breast cancer.

\section{Acknowledgments}

Funding: This work was supported by National Institute of Health [R01CA160688 to K.T.], and the National Cancer Institute (NCI) [P30CA016056] involving the use of Roswell Park Cancer Institute's Resources.

\section{Footnote}

Reporting checklist: The authors have completed the MDAR reporting checklist. Available at http://dx.doi.org/10.21037/ gs-20-571

Peer Review File: Available at http://dx.doi.org/10.21037/gs20-571

Conflicts of Interest: All authors have completed the ICMJE uniform disclosure form (available at http://dx.doi. org/10.21037/gs-20-571) Kazuaki Takabe serves as an unpaid editorial board member of Gland Surgery from March 2018 to February 2023. Dr. Yoshida reports grants and personal fees from Taiho Pharm, Asahi Kasei Pharma, Chugai Pharm., Covidien Japan, Daiichi Sankyo, Eisai, Eli Lilly Japan, Johnson \& Johnson, MerkSerono, MSD, Nippon Kayaku, Novartis, Ono Pharm., Otsuka Pharm., 
Sanofi, Takeda Pharm., Tsumura, Yakult Honsha; grants from Abbott, Abbvie, Astellas, Biogen Japan, Celgene, GlaxoSmithKline, KCI, Koninklijke Philips, Kyowa Kirin, Meiji Seika Pharma, Toray Medical,; personal fees from AstraZeneka, Bristol-Myers Squibb, Denka, EA Pharma, Olympus, Pfizer, Sanwa Kagaku Kenkyusho, SBI Pharma, Teijin Phamra, TERUMO, outside the submitted work. The other authors have no conflicts of interest to declare.

Ethical Statement: The authors are accountable for all aspects of the work in ensuring that questions related to the accuracy or integrity of any part of the work are appropriately investigated and resolved.

Open Access Statement: This is an Open Access article distributed in accordance with the Creative Commons Attribution-NonCommercial-NoDerivs 4.0 International License (CC BY-NC-ND 4.0), which permits the noncommercial replication and distribution of the article with the strict proviso that no changes or edits are made and the original work is properly cited (including links to both the formal publication through the relevant DOI and the license). See: https://creativecommons.org/licenses/by-nc-nd/4.0/.

\section{References}

1. Schreiber RD, Old LJ, Smyth MJ. Cancer immunoediting: integrating immunity's roles in cancer suppression and promotion. Science 2011;331:1565-70.

2. DeNardo DG, Coussens LM. Inflammation and breast cancer. Balancing immune response: crosstalk between adaptive and innate immune cells during breast cancer progression. Breast Cancer Res 2007;9:212.

3. Barcellos-Hoff MH, Akhurst RJ. Transforming growth factor-beta in breast cancer: too much, too late. Breast Cancer Res 2009;11:202.

4. van Diest PJ, van der Wall E, Baak JPA. Prognostic value of proliferation in invasive breast cancer: a review. J Clin Pathol 2004;57:675-81.

5. Aleskandarany MA, Green AR, Benhasouna AA, et al. Prognostic value of proliferation assay in the luminal, HER2-positive, and triple-negative biologic classes of breast cancer. Breast Cancer Res 2012;14:R3.

6. von Minckwitz G, Untch M, Blohmer JU, et al. Definition and Impact of Pathologic Complete Response on Prognosis After Neoadjuvant Chemotherapy in Various Intrinsic Breast Cancer Subtypes. J Clin Oncol 2012;30:1796-804.
7. Katsuta E, Maawy AA, Yan L, et al. High expression of bone morphogenetic protein (BMP) 6 and BMP7 are associated with higher immune cell infiltration and better survival in estrogen receptor-positive breast cancer. Oncol Rep 2019;42:1413-21.

8. Katsuta E, Qi Q, Peng X, et al. Pancreatic adenocarcinomas with mature blood vessels have better overall survival. Sci Rep 2019;9:1310.

9. McDonald KA, Kawaguchi T, Qi Q, et al. Tumor Heterogeneity Correlates with Less Immune Response and Worse Survival in Breast Cancer Patients. Ann Surg Oncol 2019;26:2191-9.

10. Narayanan S, Kawaguchi T, Yan L, et al. Cytolytic Activity Score to Assess Anticancer Immunity in Colorectal Cancer. Ann Surg Oncol 2018;25:2323-31.

11. Okano M, Oshi M, Butash AL, et al. Triple-Negative Breast Cancer with High Levels of Annexin A1 Expression Is Associated with Mast Cell Infiltration, Inflammation, and Angiogenesis. Int J Mol Sci 2019;20:4197.

12. Takeshita T, Asaoka M, Katsuta E, et al. High expression of polo-like kinase 1 is associated with TP53 inactivation, DNA repair deficiency, and worse prognosis in ER positive Her2 negative breast cancer. American journal of translational research 2019;11:6507-21.

13. Tokumaru Y, Katsuta E, Oshi M, et al. High Expression of miR-34a Associated with Less Aggressive Cancer Biology but Not with Survival in Breast Cancer. Int J Mol Sci 2020;21:3045.

14. Tokumaru Y, Oshi M, Katsuta E, et al. KRAS signaling enriched triple negative breast cancer is associated with favorable tumor immune microenvironment and better survival. Am J Cancer Res 2020;10:897-907.

15. Asaoka M, Ishikawa T, Takabe K, et al. APOBEC3Mediated RNA Editing in Breast Cancer is Associated with Heightened Immune Activity and Improved Survival. Int J Mol Sci 2019;20:5621.

16. Katsuta E, Yan L, Takeshita T, et al. High MYC mRNA Expression Is More Clinically Relevant than MYC DNA Amplification in Triple-Negative Breast Cancer. Int J Mol Sci 2019;21:217.

17. Kawaguchi T, Yan L, Qi Q, et al. Overexpression of suppressive microRNAs, miR-30a and miR-200c are associated with improved survival of breast cancer patients. Sci Rep 2017;7:15945.

18. Oshi M, Takahashi H, Tokumaru Y, et al. G2M Cell Cycle Pathway Score as a Prognostic Biomarker of Metastasis in Estrogen Receptor (ER)-Positive Breast Cancer. Int J Mol Sci 2020;21:2921. 
19. Ramanathan R, Olex AL, Dozmorov M, et al. Angiopoietin pathway gene expression associated with poor breast cancer survival. Breast Cancer Res Treat 2017;162:191-8.

20. Sato S, Nagahashi M, Koike T, et al. Impact of Concurrent Genomic Alterations Detected by Comprehensive Genomic Sequencing on Clinical Outcomes in East-Asian Patients with EGFR-Mutated Lung Adenocarcinoma. Sci Rep 2018;8:1005.

21. Tokumaru Y, Asaoka M, Oshi M, et al. High Expression of microRNA-143 is Associated with Favorable Tumor Immune Microenvironment and Better Survival in Estrogen Receptor Positive Breast Cancer. Int J Mol Sci 2020;21:3213.

22. Thorsson V, Gibbs DL, Brown SD, et al. The Immune Landscape of Cancer. Immunity 2018;48:812-30.e14.

23. Newman AM, Liu CL, Green MR, et al. Robust enumeration of cell subsets from tissue expression profiles. Nat Methods 2015;12:453-7.

24. Asaoka M, Patnaik SK, Zhang F, et al. Lymphovascular invasion in breast cancer is associated with gene expression signatures of cell proliferation but not lymphangiogenesis or immune response. Breast Cancer Res Treat 2020;181:309-22.

25. Narayanan S, Kawaguchi T, Peng X, et al. Tumor Infiltrating Lymphocytes and Macrophages Improve Survival in Microsatellite Unstable Colorectal Cancer. Sci Rep 2019;9:13455.

26. Okano M, Oshi M, Butash AL, et al. Estrogen Receptor Positive Breast Cancer with High Expression of Androgen Receptor has Less Cytolytic Activity and Worse Response to Neoadjuvant Chemotherapy but Better Survival. Int J Mol Sci 2019;20:2655.

27. Oshi M, Okano M, Maiti A, et al. Novel Breast Cancer

Cite this article as: Le L, Tokumaru Y, Oshi M, Asaoka M, Yan L, Endo I, Ishikawa T, Futamura M, Yoshida K, Takabe $\mathrm{K}$. Th2 cell infiltrations predict neoadjuvant chemotherapy response of estrogen receptor-positive breast cancer. Gland Surg 2021;10(1):154-165. doi: 10.21037/gs-20-571
Brain Metastasis Patient-Derived Orthotopic Xenograft Model for Preclinical Studies. Cancers (Basel) 2020;12:444.

28. Young J, Kawaguchi T, Yan L, et al. Tamoxifen sensitivityrelated microRNA-342 is a useful biomarker for breast cancer survival. Oncotarget 2017;8:99978-89.

29. Kaewkangsadan V, Verma C, Eremin JM, et al. Crucial Contributions by T Lymphocytes (Effector, Regulatory, and Checkpoint Inhibitor) and Cytokines (TH1, TH2, and TH17) to a Pathological Complete Response Induced by Neoadjuvant Chemotherapy in Women with Breast Cancer. J Immunol Res 2016;2016:4757405.

30. Ragab HM, Samy N, Afify M, et al. Assessment of Ki-67 as a potential biomarker in patients with breast cancer. J Genet Eng Biotechnol 2018;16:479-84.

31. Becht E, Giraldo NA, Germain C, et al. Immune Contexture, Immunoscore, and Malignant Cell Molecular Subgroups for Prognostic and Theranostic Classifications of Cancers. Adv Immunol 2016;130:95-190.

32. Mills CD, Lenz LL, Harris RA. A Breakthrough: Macrophage-Directed Cancer Immunotherapy. Cancer Res 2016;76:513-6.

33. Schiavoni G, Gabriele L, Mattei F. The tumor microenvironment: a pitch for multiple players. Front Oncol 2013;3:90.

34. Oshi M, Takahashi H, Tokumaru Y, et al. The E2F Pathway Score as a Predictive Biomarker of Response to Neoadjuvant Therapy in ER+/HER2- Breast Cancer. Cells 2020;9:1643.

35. Tokumaru Y, Oshi M, Katsuta E, et al. Intratumoral Adipocyte-High Breast Cancer Enrich for Metastatic and Inflammation-Related Pathways but Associated with Less Cancer Cell Proliferation. Int J Mol Sci 2020;21:5744. 


\section{Supplementary}

Table S1 Demographic and clinicopathological factors of Th2 High and Th2 low groups in TCGA breast cancer cohort

\begin{tabular}{|c|c|c|c|}
\hline \multirow{2}{*}{ Clinicopathological factor } & \multicolumn{3}{|c|}{ Whole cohort $(n=1,069)$} \\
\hline & Th2 High, n=535 & Th2 Low, $n=534$ & $P$ value \\
\hline Age & & & 0.235 \\
\hline$<65 y$ & 378 & 359 & \\
\hline$\geq 65 y$ & 157 & 175 & \\
\hline Stage & & & $<0.01$ \\
\hline 1 & 72 & 106 & \\
\hline 2 & 328 & 277 & \\
\hline 3 & 117 & 127 & \\
\hline 4 & 8 & 10 & \\
\hline Unknown & 10 & 14 & \\
\hline T category & & & $<0.05$ \\
\hline 1 & 120 & 151 & \\
\hline 2 & 333 & 282 & \\
\hline 3 & 58 & 78 & \\
\hline 4 & 20 & 18 & \\
\hline Unknown & 4 & 5 & \\
\hline $\mathrm{N}$ category & & & $<0.01$ \\
\hline 0 & 248 & 256 & \\
\hline 1 & 183 & 163 & \\
\hline 2 & 69 & 49 & \\
\hline 3 & 25 & 50 & \\
\hline Unknown & 10 & 16 & \\
\hline M category & & & 0.822 \\
\hline 0 & 478 & 407 & \\
\hline 1 & 10 & 10 & \\
\hline Unknown & 47 & & \\
\hline ER status & & & 0.0879 \\
\hline Negative & 128 & 108 & \\
\hline Positive & 374 & 408 & \\
\hline Unknown & 33 & 18 & \\
\hline PgR status & & & 0.183 \\
\hline Negative & 177 & 161 & \\
\hline Positive & 324 & 353 & \\
\hline Unknown & 34 & 20 & \\
\hline HER2 status & & & $<0.05$ \\
\hline Negative & 265 & 283 & \\
\hline Positive & 92 & 66 & \\
\hline Unknown & 178 & 185 & \\
\hline Histologic subtype & & & $<0.001$ \\
\hline Infiltrating ductal carcinoma & 442 & 321 & \\
\hline Infiltrating lobular carcinoma & 48 & 153 & \\
\hline Other & 29 & 45 & \\
\hline Unknown & 16 & 15 & \\
\hline
\end{tabular}



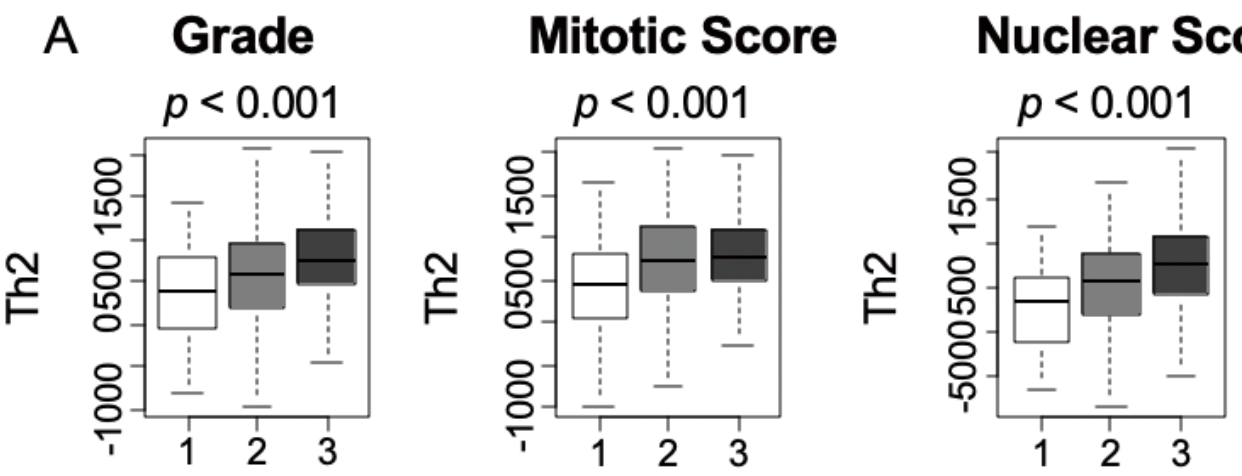

Tubular score

B

Ki-67

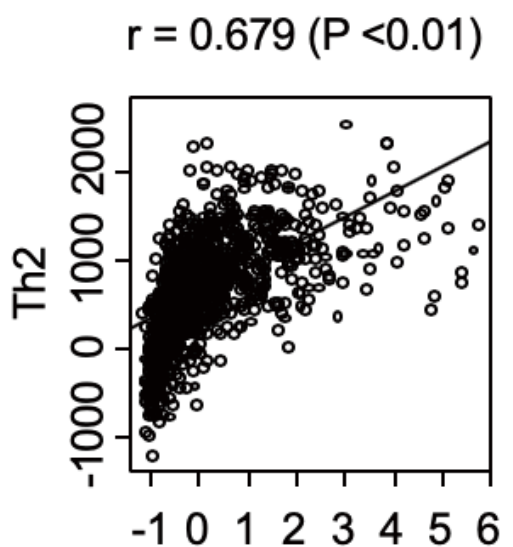

Proliferation

$$
r=0.676(P<0.01)
$$

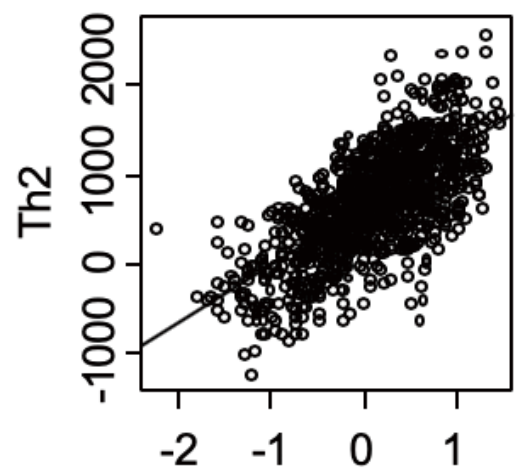

Figure S1 The association between Th2 expression levels, cancer staging, pathological grade, and proliferation in the whole cohort. (A) Analysis with the whole cohort showed that Th2 levels have a significant correlation with pathological grade, especially high mitotic and nuclear score $(\mathrm{P}<0.001)$. (B) Whole cohort showed high correlation of Th2 levels with Ki-67 and proliferation score ( $\mathrm{r}=0.679$ and $\mathrm{r}=0.676$, respectively). 


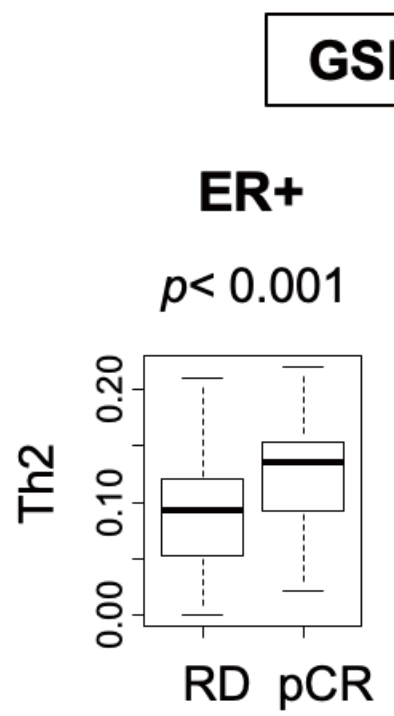

ER-

$p<0.084$

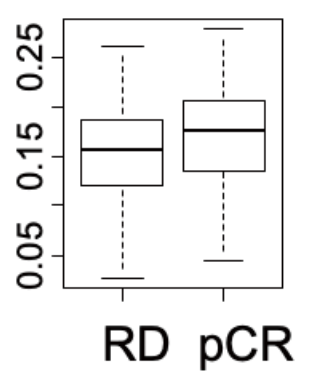

GSE23988

ER+

ER-

$p=0.385$

ER-
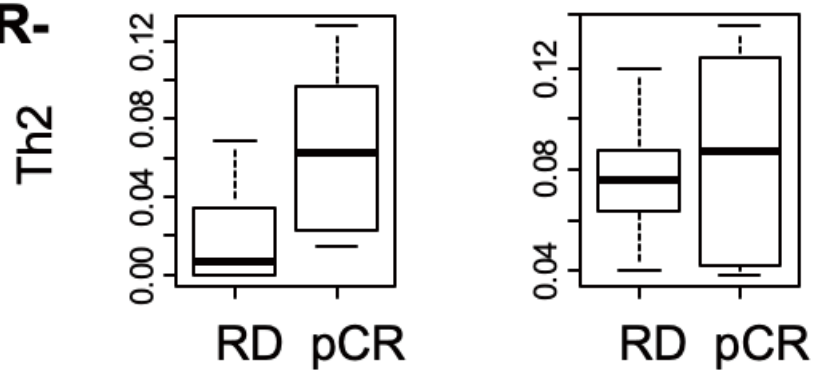

Figure S2 Patients who achieved pCR have significantly higher Th2 levels compared with the ones who had residual disease (RD) in analysis of two cohorts (GSE25066 and GSE23988). 